\title{
V7 - BRAZILIAN MENINGOCOCCAL C CONJUGATE VACCINE: SCALING UP STUDIES
}

$\underline{\text { Renata Chagas Bastos }}^{1}$, Milton Neto da Silva ${ }^{1}$, Iaralice Medeiros de Souza ${ }^{1}$, Rafael Ficha Alves $^{2}$, José Godinho da Silva Junior ${ }^{1}$, Ricardo de Andrade Medronho ${ }^{3}$, Ivna Alana Freitas Brasileiro da Silveira ${ }^{1}$

${ }^{1}$ Bio-Manguinhos, Oswaldo Cruz Foundation, Technological Development, Rio de Janeiro, Brazil

${ }^{2}$ Bio-Manguinhos, Oswaldo Cruz Foundation, Bacterial Vaccine Department, Rio de Janeiro, Brazil

${ }^{3}$ Chemical Engeneering Department, Federal University of Rio de Janeiro, Center of Technology, Rio de Janeiro, Brazil

Objectives: This work is related to comparative analysis between obtained data from pilot and industrial scale of the meningococcal $\mathrm{C}$ conjugate vaccine under GMP.

Methods: Some relevant parameters were used to compare differences/similarities between pilot and industrial scales referred to meningococcal $\mathrm{C}$ conjugate bulk (MPCT) production. Such parameters were: time spent in the production process; processing losses during purification by tangential flow filtration and the polysaccharide to protein ratio (PS/Prot) in final conjugate. Data from fifteen batches of oxidized meningococcal C polysaccharide (MPCO), 9 batches of hidrazide-activated monomeric tetanus toxoid (MATT) and 4 batches of MPCT obtained in pilot scale were compared with scale up data concerned to 5 batches of MPCO, 9 batches of MATT and 3 batches of MPCT. SEC chromatographic profile, ${ }^{1} \mathrm{HNMR}$ spectrum and content of free polysaccharide by capillary zone electrophoresis (CZE) were also used to provide comparison between both scale processes.

Results: Five-fold increase in reaction and purification scale of the MPCO, allowed reaching a final volume eight times greater with final product concentration close to the observed in pilot scale, practically spending the same pilot scale time. Such modification 
gave sufficient amount of MPCO for two batches of conjugation reaction from only one batch of oxidized product. During the scale up: in the MPCO purification step it was observed a minor loss $(16 \%)$ compared to the pilot scale process; the 2.5-fold increase in the reaction and purification of the MATT resulted in 20 minutes increasing, without significant losses; the five-fold increase in MPCT reaction and purification has shown the same PS/Prot with a small increase of 20 minutes in the total processing time compared with pilot scale. The preliminary data related to quality control assays (SEC, ${ }^{1} \mathrm{HNMR}$ and CZE) indicated efficiency of scale up purification process by tangential flow filtration in the removal of reagents and by-products during the purification of MPCO and MATT. In addition the purification process was able to minimize unbound polysaccharide in the conjugate bulks. Moreover the SEC chromatographic profiles and ${ }^{1} \mathrm{HRMN}$ spectra were similar for the products obtained in both scales. Conjugate production was clearly demonstrated by disappearance of the aldehyde groups assignments observed in analysis of MPCO. All MPCT batches in pilot and industrial scale exhibited sugar free contents below $10 \%$ as shown by CZE analysis.

Conclusion: The scaling up of all processes involved in the production of meningococcal C conjugate aiming vaccine preparation was considered relevant and the physicochemical characteristics of the molecules (MPCO, MATT, MPCT) related to studied scales were similar. These results demonstrated the industrial plant capacity of Bio-Manguinhos to supply annual demand of vaccines to the National Program of Immunization, after conducting phase III clinical trials. 